\title{
TIPO DE ALIMENTO, SOBREVIVÊNCIA E DESEMPENHO INICIAL DE PÓS-LARVAS DE PACU (Piaractus mesopotamicus)
}

\author{
Types of preys on growth and survival of pacu (Piaractus mesopotamicus) pos-larvae
}

\author{
Martha Janeth Prieto ${ }^{1}$, Priscila Vieira Rosa Logato ${ }^{2}$, Gilson Ferreira de Moraes ${ }^{1}$, \\ Daniel Okamura ${ }^{3}$, Felipe Guedes de Araújo ${ }^{3}$
}

\begin{abstract}
RESUMO
Conduziu-se este trabalho com o objetivo de avaliar a viabilidade técnica do uso de zooplâncton enriquecido na alimentação de pós-larvas de pacu (Piaractus mesopotamicus). Na Estação Ambiental de Itutinga, da Companhia Energética de Minas Gerais (CEMIG), foi realizada, durante 5 dias, a larvicultura de pacu, usando 30 caixas plásticas com capacidade de 30 litros de água e renovação contínua, em uma densidade de 10 pós-larvas por litro; foram avaliados 6 tratamentos de alimentação: zooplâncton sem enriquecimento, zooplâncton com 4 diferentes níveis de enriquecimento $(0,1 ; 0,5 ; 1,0 ; 1,5 \mathrm{~g}$ de óleo de peixe) e náuplios de artêmia, em um delineamento em blocos casualizados com 6 tratamentos e 5 repetições. Foram avaliados os parâmetros de desempenho nas póslarvas, comprimento, peso, sobrevivência e resistência ao estresse. Os resultados demonstraram que o uso de zooplâncton enriquecido é viável na larvicultura de pacu, permitindo adequado desempenho das pós-larvas quanto ao comprimento, à sobrevivência e à resistência ao estresse.
\end{abstract}

Termos para indexação: Aqüicultura, nutrição, alimento vivo, zooplâncton, larvicultura, pacu.

\begin{abstract}
The objective of this study was to evaluate the technical viability of the ooplankton enriched used in the feeding of pacu (Piaractus mesopotamicus) pos-larvaes. In the Environmental Station of Itutinga of the Energy Company of Minas Gerais (CEMIG) this experiment was developmented, for 5 days last, the pacu larvicultura, by using 30 plastic boxes with capacity of 30 liters of water and continuous renewal, in a density of 10 powder-larvas per liter; they were appraised 6 feeding treatments: ooplankton without enrichment, ooplankton with 4 different enrichment levels $(0,1 ; 0,5 ; 1,0 ; 1,5 \mathrm{~g}$ of fish oil) and artêmia náuplios, in a randomly blocks design with 6 treatments and 5 repetitions. The performance of larvaes as well as length, weight, survival and resistance to the stress were evaluated. The results shown that the ooplankton enriched is viable in the pacu (Piaractus mesopotamicus) pos-larvaes performance in terms of length, survival and the resistance to the stress.
\end{abstract}

Index terms: Aquaculture, nutrition, live food, zooplankton, fish larvae, pacu.

(Recebido para publicação em 29 de janeiro de 2004 e aprovado em 7 de julho de 2005)

\section{INTRODUÇÃO}

A piscicultura no Brasil, ainda apresenta resultados modestos de desenvolvimento devido aos processos de produção adotados e à falta de informações sobre as espécies nativas com potencial zootécnico. O ponto crítico na vida dos peixes é quando a larva se alimenta, neste ponto inicia o estágio de pós-larva; além das necessidades ambientais, a pós-larva requer alimentos externos apropriados, tanto qualitativamente quanto quantitativamente. A alimentação e a nutrição têm sido apontadas como os principais fatores responsáveis pelos freqüentes insucessos da larvicultura (JOMORI, 2001; PORTELLA et al., 2002; TESSER, 2002).

As pós-larvas de pacu são consideradas altriciais. O fornecimento e disponibilidade de alimento vivo são fundamentais na alimentação inicial de pós-larvas altriciais
(PORTELLA et al., 2002). Essas pós-larvas apresentam pouca reserva de vitelo e o trato digestivo indiferenciado, utilizando enzimas das presas ingeridas (zooplâncton), para facilitar seu processo de digestão, sendo dependentes das mesmas enquanto desenvolvem seu próprio sistema digestório (SIPAÚBA-TAVARES \& ROCHA, 2003). O zooplâncton é a principal fonte de proteínas, aminoácidos livres e ácidos graxos essenciais ao desenvolvimento inicial das pós-larvas (PORTELLA et al., 2002; SIPAÚBATAVARES \& ROCHA, 2003).

No desenvolvimento inicial das larvas e pós-larvas de peixes, destacam-se, dentre os requerimentos nutricionais, os ácidos graxos essenciais (AGE) constituídos pelo ácido linolênico (LNA, 18:3n-3), linoléico (LA, 18:2 n-6) e araquidônico (AA, 20:4 n-6). Os AGE participam da formação dos fosfolipídeos da membrana celular, sendo responsáveis na manutenção da integridade,

Mestre em Zootecnia pela Universidade Federal de Lavras.

2 Doutora em Zootecnia, Professora Adjunta do Departamento de Zootecnia da Universidade Federal de Lavras/UFLA - Cx. P. 3037 - $37200-000$ Lavras, MG - priscila@ufla.br

${ }^{3}$ Alunos de graduação Curso de Zootecnia, Universidade Federal de Lavras/UFLA - Cx. P. 3037 - 37200-000 - Lavras, MG. 
fluidez e permeabilidade da célula na maioria dos tecidos (SARGENT et al., 1999) e, portanto, pelo funcionamento total do organismo. O requerimento de ácidos graxos para a construção e renovação de membranas é especialmente alto durante o rápido crescimento dos estágios de larvas e pós-larvas dos peixes. As espécies de peixes de água doce podem se alimentar de organismos planctônicos com elevado conteúdo de ácido linoléico, pois têm a capacidade de sintetizar, por dessaturação, os ácidos graxos ômega-3, principalmente o EPA, para suprir seus requerimentos (COPEMAN et al., 2002; KOVEN et al., 2001; SARGENT et al., 1999).

O conteúdo de EPA e DHA nas partículas que servem como alimento para pós-larvas de diversas espécies de peixes é importante em relação à sobrevivência das póslarvas, seu adequado crescimento e susceptibilidade ao estresse (AWAISS et al., 1996; KOLVOVSKI et al., 2000; KOVEN et al., 2001; COPEMAN et al., 2002; SARGENT et al., 1999). Por isso, para atender estes requerimentos nutricionais em pós-larvas altriciais são oferecidos alimentos vivos enriquecidos, sendo o enriquecimento um importante método que pode ser usado para transferir toda classe de elementos essenciais através dos organismos zooplanctônicos.

Mesmo com as informações já disponíveis sobre as larvas da espécie Pacu (JOMORI, 1999, 2001; TESSER et al., 2002) muitos aspectos relacionados à alimentação durante a larvicultura ainda precisam ser estudados e solucionados, visando maximizar a disponibilidade de alevinos para o mercado. Deste modo, com o presente estudo, avaliou-se a viabilidade do uso de zooplâncton enriquecido com óleo de peixe sobre a sobrevivência e o desempenho das pós-larvas de pacu.

\section{MATERIAL E MÉTODOS}

$\mathrm{Na}$ Estação Ambiental de Itutinga, Companhia Energética de Minas Gerais (CEMIG), realizou-se a larvicultura de pacu. As larvas foram obtidas de desovas induzidas, com eclosão em incubadoras cilíndricas de 100 litros, onde permaneceram por dois dias para completarem o período larval, até o início da alimentação exógena. Para a larvicultura foram utilizadas 30 caixas plásticas, cada uma com 30 litros de água com renovação contínua, onde as pós-larvas foram mantidas durante 5 dias, sob temperatura constante de $27^{\circ} \mathrm{C}$, mantida mediante o uso de termostato com aquecedor na caixa d'água. $\mathrm{O}$ valor médio de $\mathrm{pH}$ registrou-se em 6,7 e o oxigênio manteve-se em 6,53 mg/L.

Cada caixa recebeu 10 pós-larvas por litro, totalizando 300 pós-larvas/caixa. As pós-larvas foram alimentadas 3 vezes ao dia, nos horários de 8:00, 16:00 e 24:00 horas. Foram testados 6 tratamentos na alimentação das pós-larvas, cada qual com 5 repetições, num delineamento em blocos casualizados (DBC), totalizando 30 parcelas. Os 6 tratamentos foram: $\mathrm{T} 1=$ plâncton com 0,1g de óleo; $\mathrm{T} 2$ = plâncton com $0,5 \mathrm{~g}$ de óleo; $\mathrm{T} 3$ = plâncton com 1,0g de óleo; T4 = plâncton com 1,5g de óleo; T5 = plâncton sem enriquecimento e T6 = náuplios de artêmia.

O zooplâncton, composto por $63 \%$ de cladóceros e $37 \%$ de copépodos, na faixa entre $100-350 \mathrm{~mm}$, foi cultivado a partir de cepas dos gêneros Bosmia, Bosminopsis, Moina, Diaphanosoma, Cyclops, Argirodiaptomus e Calanus. O enriquecimento durante 6 horas, realizou-se com emulsão elaborada a partir de uma mistura de $20 \%$ de óleo de peixe, $7 \%$ de lecitina e $73 \%$ de água destilada. O perfil de ácidos graxos presente nos tratamentos alimentares foi avaliado mediante análise cromatográfica.

Amostras de 30 pós-larvas foram coletadas ao início e após o período experimental de 5 dias. As amostras foram armazenadas e identificadas em frascos contendo formol a $10 \%$, para posterior análise de desempenho. Nas pós-larvas coletadas foram medidos o comprimento total e o peso médio das pós-larvas ao início e final do experimento.

Com uma lente ocular micrométrica graduada num microscópio óptico com aumento de 4x. (Studar Lab.), mediram-se individualmente as longitudes totais de 30 póslarvas, desde a ponta do focinho até o final da nadadeira caudal. Para determinar o peso, as pós-larvas foram colocadas sobre papel absorvente, eliminando o excesso de umidade, sendo pesadas individualmente em uma balança analítica (Núcleo - PR 330), com precisão de 0,1mg. Com as longitudes e os pesos totais de cada unidade experimental foram estimados o ganho em peso $(\mathrm{GP}(\mathrm{mg})=$ Pmf - Pmi $)$ e comprimento $(\mathrm{GL}(\mathrm{mm})=\mathrm{Ltf}-\mathrm{Lti})$ para cada tratamento, sendo: Pmf - peso médio final; Pmi - peso médio inicial; Lti - comprimento médio total inicial e Ltf comprimento médio total final.

Ao início e final do estudo, para cada unidade experimental, foram contadas manualmente as pós-larvas e calculou-se o percentual de sobrevivência empregando a equação: $\mathrm{S}(\%)$ = (número final de pós-larvas/número inicial de pós-larvas) x 100. Igualmente, foram coletadas manualmente ao acaso 15 pós-larvas por caixa, que foram submetidas à prova de resistência ao estresse. As pós-larvas foram capturadas e colocadas em papel absorvente durante quatro minutos; posteriormente transferidas para um recipiente com água da respectiva caixa; quinze minutos depois foram contadas as que permaneceram vivas. Calculou-se a taxa de resistência ao estresse em porcentagem. 
As variáveis resposta na larvicultura de pacu foram os parâmetros de desempenho: sobrevivência, comprimento, peso e resistência ao estresse. As análises estatísticas foram realizadas pelo programa SAEG (Sistema para Análises Estatísticas e Genéticas), proposto por Jomori (1999), aplicando-se análise de variância (ANAVA). O efeito dos níveis das variáveis resposta na larvicultura foi avaliado mediante um modelo de regressão, com contrastes de médias, utilizando-se o teste de Scott-Knott, a 5\% de significância.

\section{RESULTADOS E DISCUSSÃO}

A utilização de zooplâncton enriquecido ou não com diferentes níveis de óleo na alimentação das pós-larvas de pacu (Piaractus mesopotamicus) permitiu o crescimento dos peixes em todos os tratamentos (Figura 1).

Na Tabela 1, estão apresentados os resultados das médias obtidas e do desvio padrão para o desempenho das pós-larvas nas variáveis: comprimento, peso e sobrevivência.

Em relação ao comprimento das pós-larvas, não houve diferença significativa entre os tratamentos $(p>0,05)$, pelo teste Scott-Knott (Tabela 1), registrando-se comprimentos entre 5959,00 $\pm 68,87 \mu \mathrm{m}$ e $6179,48 \pm 35,53 \mu \mathrm{m}$. As pós-larvas alimentadas com o zooplâncton em diferentes níveis de enriquecimento não apresentaram diferença significativa no comprimento, quando comparadas com as pós-larvas alimentadas com artêmia, que é o alimento geralmente usado na larvicultura destas espécies de peixes.

Os resultados do comprimento em pacu estão de acordo com os resultados obtidos por Tesser (2002) que, trabalhando na larvicultura de pacu, registrou aos 6 dias comprimentos significativamente diferentes entre 5,41 e 6,63 $\mathrm{mm}$, dependendo do tratamento alimentar, sendo dieta microencapsulada e náuplios de artêmia, respectivamente. Para a mesma espécie, Beerli (2002) reportou comprimentos significativamente diferentes aos 6 dias de idade entre 6,21 e $6,39 \mathrm{~mm}$, quando alimentadas com $100 \%$ de ração e proporção 1:1 artêmia:plâncton, respectivamente. Da mesma forma, quando alimentadas com artêmia as pós-larvas de pacu registraram comprimento de $6,74 \mathrm{~mm}$, aos seis dias depois de iniciada a alimentação exógena (JOMORI, 1999, 2001).

Com relação ao peso, pode-se observar na Tabela 1 que, houve diferença significativa entre os diferentes tratamentos $(\mathrm{p}<0,01)$. O tratamento com artêmia apresentou $1,29278 \pm 0,150 \mathrm{mg}$, sendo superior aos demais tratamentos, os quais não tiveram diferença significativa entre si. Os diferentes níveis de enriquecimento do plâncton estatisticamente não apresentam efeito sobre a variável peso nas pós-larvas de pacu (Tabela 1). Assim, é igualmente importante destacar que no tratamento correspondente ao plâncton não enriquecido não houve diferença significativa de seu efeito no peso das pós-larvas, quando comparado ao plâncton enriquecido (Tabela 1).

Os resultados do peso para o pacu concordam com o descrito por Jomori (1999), que reportou 1,50 mg, alimentandoo com náuplios de artêmia. Jamori (2001), registrou peso de $1 \mathrm{mg}$ para a mesma espécie com o mesmo tratamento. Já Beerli (2002), tratando larvas de pacu com diferentes tratamentos alimentares, obteve pesos entre 0,8 e $1,130 \mathrm{mg}$, quando alimentou-as somente com ração e proporção $1: 1$ artêmia:plâncton, respectivamente. Tesser (2002) reportou 0,4 mg e 1,4 mg para o peso das pós-larvas de pacu quando mantidas em jejum e tratadas com artêmia, respectivamente.

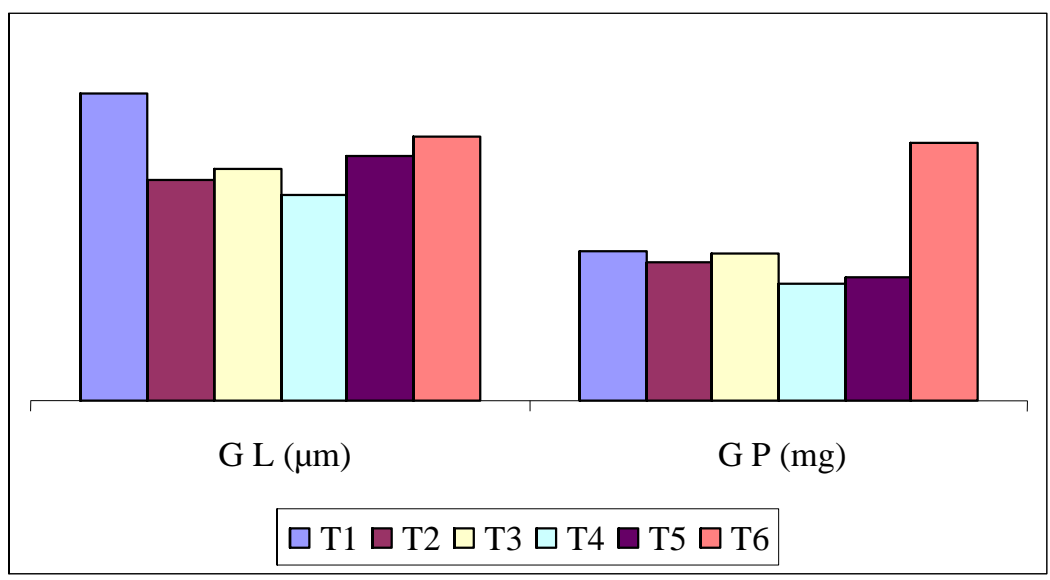

FIGURA 1 - Média dos valores de ganho em comprimento (GC) e ganho de peso (GP) das pós-larvas de pacu após o período de larvicultura. T1-T6: tratamentos de alimentação.

Ciênc. agrotec., Lavras, v. 30, n. 5, p. 1002-1007, set./out., 2006 
TABELA 1 - Parâmetros de desempenho do pacu (Piaractus mesopotamicus). Médias e desvio padrão do comprimento $(\mu \mathrm{m})$ e peso $(\mathrm{mg})$ das pós-larvas e sobrevivência $(\%)$.

\begin{tabular}{|c|c|c|c|c|}
\hline Tratamento & $\begin{array}{c}\text { Comprimento } \\
(\mu \mathrm{m})\end{array}$ & $\begin{array}{l}\text { Peso ** } \\
\text { (mg) }\end{array}$ & $\begin{array}{c}\text { Resistência ao } \\
\text { estresse (\%) }\end{array}$ & $\begin{array}{c}\text { Sobrevivência } \\
(\%)\end{array}$ \\
\hline Zooplâncton & $6044,55^{a}$ & $0,99958^{b}$ & $77,33^{\mathrm{a}}$ & $93,398^{\mathrm{a}}$ \\
\hline $0,0 \mathrm{~g}(\mathrm{~T} 5)$ & $\pm 105,38$ & $\pm 0,100$ & $\pm 22,90$ & $\pm 8,58$ \\
\hline Zooplâncton & $6179,48^{a}$ & $1,05788^{\mathrm{b}}$ & $82,66^{a}$ & $94,932^{\mathrm{a}}$ \\
\hline $0,1 \mathrm{~g}(\mathrm{~T} 1)$ & $\pm 35,53$ & $\pm 0,037$ & $\pm 28,12$ & $\pm 5,76$ \\
\hline Zooplâncton & $6083,48^{a}$ & $1,05385^{\mathrm{b}}$ & $95,00^{\text {a }}$ & $89,320^{a}$ \\
\hline $0,5 \mathrm{~g}(\mathrm{~T} 2)$ & $\pm 211,97$ & $\pm 0,067$ & $\pm 15,3$ & $\pm 7,28$ \\
\hline Zooplâncton & $6031,34^{\mathrm{a}}$ & $1,05195^{\mathrm{b}}$ & $81,66^{a}$ & $94,240^{\mathrm{a}}$ \\
\hline $1,0 \mathrm{~g}(\mathrm{~T} 3)$ & $\pm 97,63$ & $\pm 0,048$ & $\pm 17,89$ & $\pm 6,87$ \\
\hline Zooplâncton & $5959,00^{a}$ & $0,98836^{\mathrm{b}}$ & $88,00^{\text {a }}$ & $86,598^{a}$ \\
\hline $1,5 \mathrm{~g}(\mathrm{~T} 4)$ & $\pm 68,87$ & $\pm 0,057$ & $\pm 20,22$ & $\pm 25,59$ \\
\hline Artêmia & $6087,27^{\mathrm{a}}$ & $1,29278^{\mathrm{a}}$ & $84,00^{\text {a }}$ & $90,196^{a}$ \\
\hline (T6) & $\pm 80,38$ & $\pm 0,150$ & $\pm 15,35$ & $\pm 6,37$ \\
\hline
\end{tabular}

* Significativo $(\mathrm{P}<0,05) \quad * *$ Significativo $(\mathrm{P}<0,01)$;

Médias com letras diferentes nas colunas diferem entre si pelo teste de Scott-Knott.

O zooplâncton, enriquecido ou não, apresenta de $6,86 \%$ a $14,34 \%$ de ácido linoléico; $12,63 \%$ a $15,95 \%$ de AA; $2,51 \%$ a $6,08 \%$ de DHA e de $0,43 \%$ a $0,53 \%$ de EPA. As proporções DHA/AA e AA/EPA e DHA/EPA presentes neste zooplâncton têm incidência sobre as pós-larvas estimulando seu crescimento. Atualmente, é reconhecida a importância do conteúdo de EPA e DHA nas espécies de peixes em relação à sobrevivência, adequado crescimento e resistência ao estresse das pós-larvas (AWAISS et al., 1996; COPEMAN et al., 2002; KOLVOVSKI et al., 2000; KOVEN et al., 2001; SARGENT et al., 1999).

Segundo Bell \& Sargent (2003), alimentos vivos fornecidos às pós-larvas devem oferecer um conteúdo maior que $4 \%$ de EPA em relação ao total dos ácidos graxos, podendo-se esperar uma nutrição deficiente com níveis abaixo de 3\% em EPA. O zooplâncton enriquecido nos diferentes níveis apresenta baixo conteúdo de EPA $(0,53 \%$ $-0,64 \%)$, mas alto conteúdo de ácido linolênico (18:2 n-3) $(2,94 \%-4,44 \%)$. Portanto, espera-se que o pacu, sendo uma espécie de água doce, possa sintetizar do ácido linolênico por dessaturação, os ácidos graxos ômega-3, principalmente o EPA, para suprir seus requerimentos (AWAISS et al., 1996; SARGENT et al., 1999).

No crescimento das pós-larvas é importante também a proporção DHA/EPA (COPEMAN et al., 2002; KOLVOVSKI et al., 2000; SARGENT et al., 1999). Segundo Sargent et al. (1999), a proporção adequada e recomendada de DHA/EPA é 2:1 para pós-larvas de peixes marinhos; o mesmo autor afirma, em relação às pós-larvas de peixes marinhos e de água doce, que tanto a quantidade absoluta de cada ácido graxo quanto sua relativa proporção são importantes na nutrição das pós-larvas.

Outra variável analisada foi a sobrevivência. $\mathrm{Na}$ Tabela 1, percebe-se que não houve diferença significativa $(p>0,05)$ entre os diferentes tratamentos para as médias de sobrevivência alcançadas pelas pós-larvas depois de 5 dias de larvicultura. Destaca-se o fato que em todos os tratamentos houve uma alta sobrevivência, superior a $85 \%$, sendo em quatro, dos seis tratamentos, superior a $90 \%$. Estes resultados evidenciam que o desempenho das póslarvas de pacu em relação à sobrevivência é igual ao serem alimentadas com plâncton enriquecido ou não, quando comparadas com aquelas alimentadas com artêmia, porém, o tratamento T4 apresentou-se com a menor média de sobrevivência e o maior desvio padrão.

Os resultados de sobrevivência das pós-larvas de pacu concordam com os resultados observados na larvicultura de mesma espécie por outros autores. Jomori (2001) relatou uma sobrevivência de $86,38 \%$ das pós-larvas quando alimentadas durante 6 dias com náuplios de artêmia; Tesser (2002) observou uma sobrevivência de $63,6 \%$ depois de 12 dias alimentando as pós-larvas durante 6 dias com náuplios de artêmia e 6 dias com co-alimentação artêmia + dieta microencapsulada e reportou $87,1 \%$ de sobrevivência quando alimentadas durante 12 dias somente com artêmia. 
As pós-larvas de pacu cultivadas em laboratório apresentaram altas taxas de sobrevivência, evidenciando, sob esse aspecto, a eficiência do sistema de cultivo e a viabilidade do zooplâncton como partícula alimentícia com efeitos tão positivos quanto os obtidos com artêmia.

As pós-larvas de pacu apresentaram uma alta sobrevivência depois de submetidas à prova de estresse, sendo superior a $80 \%$ na maioria dos casos (Tabela 1). Não houve diferença significativa entre os tratamentos ( $p>0,05)$. A maior média numérica de resistência ao estresse registrou-se nos tratamentos alimentados com plâncton enriquecido e o menor valor médio no tratamento T5 com $77,33 \pm 22,90 \%$, correspondente às pós-larvas alimentadas com plâncton não enriquecido (Tabela 1).

As pós-larvas de pacu aparentemente não apresentam resposta diferenciada entre tratamentos. Krieger-Azzolini et al. (1989), estudando indicadores endócrinos e metabólicos no estresse em pacu, afirmam que as espécies de peixes apresentam grande variabilidade interespecífica quanto à tolerância ao agente estressante, e as reações fisiológicas devem ser consideradas tanto no que se refere ao tipo resposta, como a caracterização do grau de tolerância de uma determinada espécie em relação ao meio ambiente no qual se encontra.

Segundo Bell \& Sargent (2003), níveis entre $1 \%$ e $2 \%$ de ácido araquidônico na dieta podem melhorar o crescimento, sobrevivência e resistência ao estresse nos peixes. Porém, pós-larvas com suplemento de AA na dieta são mais resistentes ao estresse agudo, mas apresentamse mais susceptíveis ao estresse crônico por mudanças diárias, quando comparadas àquelas alimentadas com baixos níveis de AA. No presente estudo, os níveis de AA no zooplâncton, enriquecido ou não, foi alto (>10\%) quando comparado com artêmia (3,60\%). Esses níveis não parecem afetar a resposta metabólica no pacu.

No mesmo sentido, pode-se pensar que as póslarvas estiveram submetidas ao estresse nutritivo durante a larvicultura, induzido, principalmente, por um excesso de ácidos graxos, como o 18:3 n-3 (linolênico), associado aos baixos níveis de 20:5 n-3 (EPA) (FRASCALOSSI, 1998; SARGENT, et al., 1999), quando alimentadas com artêmia. Porém, a resposta nas pós-larvas não parece estar associada a este fato.

De acordo com o exposto e os resultados deste estudo, a alimentação com plâncton, com ou sem enriquecimento, com baixas ou altas porcentagens de AA, não tem efeito direto na resposta diante do estresse para as pós-larvas de pacu. A alta resistência ao estresse registrada pelos resultados de sobrevivência em pacu, sem apresentar diferença significativa entre tratamentos, indica a condição de bem-estar das pós-larvas, como reflexo de uma adequada qualidade nutricional destes alimentos como primeira alimentação.

\section{CONCLUSÃO}

O uso de zooplâncton enriquecido ou não com óleo de peixe é viável na larvicultura do pacu (Piaractus mesopotamicus), permitindo adequado desempenho das pós-larvas quanto ao comprimento, à sobrevivência e à resistência ao estresse.

\section{REFERÊNCIAS BIBLIOGRÁFICAS}

AWAISS, A.; KESTEMONT, P.; MICHA, J. C. Fatty acid profiles of two freshwater fish larvae (gudgeon and perch) reared with Brachionus calyciflorus Pallas (rotifer) and/or dry diet. Aquaculture Research, Oxford, n. 9, p. 651-658, Sept. 1996.

BEERLI, E. L. Alimentação e comportamento de pós-larvas de pacu. 2002. 51 p. Dissertação (Mestrado) - Universidade Federal de Lavras, Lavras, 2002.

BELL, J. G.; SARGENT, J. R. Arachidonic acid in aquaculture feeds: current status and future opportunities. Aquaculture, Amsterdam, v. 218, n. 1/4, p. 491-499, Mar. 2003.

COPEMAN, L. A.; PARRISH, C. C.; BROWN, J. A.; HAREL, M. Effects of docosahexaenoic, eicosapentaenoic, and arachidonic acids on the early growth, survival, lipid composition and pigmentation of yellowtail flounder (Limanda ferruginea): a live food enrichment experiment. Aquaculture, Amsterdam, v. 210, n. 1/4, p. 285-304, July 2002.

FRACALOSSI, D. M. Doenças nutricionais em peixes. In: SIMPÓSIO SOBRE MANEJO E NUTRIÇÃO DE PEIXES, 2., 1998, Piracicaba. Anais... Campinas: CBNA, 1998. $198 \mathrm{p}$.

JOMORI, R. K. Desenvolvimento, sobrevivência e aspectos econômicos da produção de alevinos de pacu, Piaractus mesopotamicus (Holemberg, 1887), diretamente em viveiros ou com diferentes períodos de cultivo inicial de larvas em laboratório. 2001. $69 \mathrm{f}$. Dissertação (Mestrado) - Universidade Estadual Paulista, Jaboticabal, 2001. 
JOMORI, R. K. Estudo sobre a alimentação de larvas de pacu, Piaractus mesopotamicus (Holemberg, 1887), com náuplios de artemia e a sua substituição por dieta artificial. 1999. 70 f. (Trabalho de graduação) - Faculdade de Ciências Agrárias e Veterinárias, Universidade Estadual Paulista, Jaboticabal, 1999.

KOLKOVSKI, S.; CZESNY, S.; YACKEY, C.; MOREAU, R.; CIHLA, F.; MAHAN, D.; DABROWSKI, K. The efect of vitamins $C$ and $E$ in (n-3) highly unsaturated fatty acidsenriched Artemia nauplii on growth, survival, and stress resistance of fresh water walleye Stizostedion vitreum larvae. Aquaculture Nutrition, Columbus, v. 6, n. 3, p. 199206, Sept. 2000.

KOVEN, W.; BARR, Y.; LUTZKY, S.; BEN-ATIA, I.; WEISS, R.; HAREL, M.; BEHRENS, P.; TANDLER, A. The effects of dietary arachidonic acid (20:4 n-6) on growth, survival and resistance to handling stress in gilthead seabream (Sparus aurata) larvae. Aquaculture, Amsterdam, v. 193, n. 1/2, p. 107-122, Feb. 2001.

KRIEGER-AZZOLINI, M. H.; CAROOLSFELD, J.; DELATTRE, E.; CECCARELLI, P. S.; MENEZES, F. V. Determinação dos indicadores endócrinos e metabólicos no estresse de manejo em pacu juvenil, Piaractus mesopotamicus Holmberg, 1887. Boletim Técnico CEPTA, Pirassununga, v. 2, p. 35-42, 1989.

PORTELLA, M. C.; TASSER, M. B.; JOMORI, R. K.; CARNEIRO, D. J. Substituição do alimento vivo na larvicultura. In: SIMPÓSIO BRASILEIRO DE AQÜICULTURA, 12., 2002, Goiânia, GO. Anais... Goiânia: ABRAQ, 2002.

SARGENT, J.; BELL, G.; McEVOY, L.; TOCHER, D.; ESTEVEZ, A. Recent developments in the essential fatty acid nutricion of fish. Aquaculture, Amsterdam, v. 177, n. 1/4, p. 191-199, July 1999.

SIPAÚBA-TAVARES, L. H.; ROCHA, O. Produção de plâncton (Fitoplâncton e Zooplâncton) para alimentação de organismos aquáticos. São Carlos: RIMA, 2003. 106 p.

TESSER, M. B. Desenvolvimento do trato digestório e crescimento de larvas de pacu, Piaractus mesopotamicus (Holmberg, 1887) em sistemas de co-alimentação com náuplios de artemia e dieta microencapsulada. 2002. $59 \mathrm{f}$. Dissertação (Mestrado) - Universidade Estadual Paulista, Jaboticabal, 2002. 